\title{
H3K9 histone methyltransferase G9a ameliorates dilated cardiomyopathy via the downregulation of cell adhesion molecules
}

\author{
GUIYING CHEN**, XU WANG ${ }^{*}$, YINA ZHANG, XIAOXUE RU, LIJUN ZHOU and YE TIAN
}

Department of Cardiology, The First Affiliated Hospital of Harbin Medical University, Harbin, Heilongjiang 150001, P.R. China

Received February 16, 2014; Accepted November 19, 2014

DOI: $10.3892 / \mathrm{mmr} .2015 .3218$

\begin{abstract}
Dilated cardiomyopathy (DCM) is one of the leading causes of mortality; however, the underlying molecular mechanisms of DCM remain to be elucidated. H3K9 histone methyltransferase G9a has been previously characterized, although its functions in DCM are not yet understood. Cell adhesion molecules (CAM) are highly expressed in diseased human hearts and were thought to contribute to chronic degeneration in cardiac incompetence; however, it has been suggested that G9a may suppress the effects of CAM. The aim of the present study was to investigate whether G9a decreased the risk of DCM via regulation of CAM expression. A rat model of DCM was induced using furazolidone (FZ) treatment and numerous parameters were examined. G9a RNA interference (RNAi) was applied to primary neonatal cardiomyocytes (PNCs). Reverse transcription quantitative polymerase chain reaction and western blot analyses were used to examine the expression levels of G9a in the DCM model and PNCs. The growth rate of PNCs was evaluated following G9a RNAi and FZ treatment. The results confirmed that the expression levels of G9a were significantly decreased in the DCM model compared with those in the control group $(\mathrm{P}<0.01)$. Conversely, CAM expression levels were significantly increased in the DCM model compared with those in the control group $(\mathrm{P}<0.01)$. In PNCs, the expression of CAM was upregulated following G9a silencing using RNAi. Following three-day culture, the growth rate of PNCs was inhibited by 70 and $35 \%$ following FZ treatment and G9a RNAi, respectively. In conclusion, G9a ameliorated DCM via downregulation of CAMs, therefore indicating its potential for use in the treatment of DCM.
\end{abstract}

Correspondence to: Dr Lijun Zhou or Dr Ye Tian, Department of Cardiology, The First Affiliated Hospital of Harbin Medical University, 23 Youzheng Street, Harbin, Heilongjiang 150001, P.R. China

E-mail: lijunzhouhb@163.com

E-mail: yetianhb@163.com

*Contributed equally

Key words: furazolidone, animal model, dilated cardiomyopathy, H3K9 histone methyltransferase G9a, cell adhesion molecules

\section{Introduction}

Dilated cardiomyopathy (DCM) is a disease that causes injury to the heart muscle; the heart may become enlarged and weakened, therefore restricting its ability to effectively pump blood (1). In addition, DCM may affect numerous other organs, including the liver and lungs, and may even affect entire body systems. DCM is one of the leading causes of mortality in the world (2); however, the underlying molecular mechanisms of DCM remain to be elucidated.

$\mathrm{H} 3 \mathrm{~K} 9$ histone methyltransferase G9a is localized in euchromatin and catalyzes the methylation of histone (3). G9a has an important role in the silencing of numerous genes. For example, Oct-3/4 is a homeobox gene with a Pit-Oct-Unc domain, which is expressed in the process of producing cells for sexual reproduction and during the embryonic period (4); during early embryogenesis, G9a mediates the inactivation of Oct-3/4 (5). G9a has been previously characterized, although its functions in DCM are not yet understood.

G9a was reported to silence cell adhesion molecules (CAMs) in the development of lung cancer (6). CAMs exhibit pleiotropic biological effects and have been reported to be overexpressed in patients with DCM (7), suggesting an association between the CAM levels and the severity of the disease (8). CAMs are elevated in patients with heart failure and may have an important role in the pathogenesis of heart failure via mediating the cell-cell interactions of the immune response (9); therefore, G9a may reduce the progression of DCM via silencing the CAM gene, therefore reducing the enhanced levels of CAM in heart disease $(6,10)$. Furthermore, the promotion of DCM epigenetics research is essential for elucidation of the underlying molecular mechanism of DCM.

Numerous animal models are available for the study of DCM. For example, a rat model of myocarditis has been created through immunization with porcine cardiac myosin, which resulted in severe heart failure characterized by increased cardiac fibrosis and left ventricular (LV) dilation (11). In addition, furazolidone, $\mathrm{N}$-(5-nitro-2-furfurylidene)-3-amino-2-oxazolidone (FZ), is a nitrofuran that has been successfully used for the treatment of certain bacterial and protozoal infections in animals (12); however, FZ also induces CAM expression and has been successfully used for the construction of DCM models (13-15). These models provide the convenience to investigate the molecular mechanism of DCM. In the present study, the rat DCM model was established via FZ treatment. The association between G9a 
and CAM expression was then explored in order to investigate the biological function of G9a in the development of DCM.

\section{Materials and methods}

Establishment of a rat model of DCM. All protocols of the present study were approved by the animal committee of the First Affiliated Hospital of Harbin Medical University (Harbin, China). A total of $60 \mathrm{Wistar}$ rats (male/female, 1:1; aged two weeks; weight, $300 \mathrm{~g}$ ) were purchased from the Shanghai Animal Resource Center (Shanghai, China). Rats were fed a commercial diet (KeAoXieLi Feed Co., Ltd., Beijing, China) and were kept at a controlled temperature $\left(22 \pm 2^{\circ} \mathrm{C}\right)$ in a $12-\mathrm{h}$ light/dark cycle. Rats were divided into a control and a DCM group. Rats in the DCM group were administered $20 \mathrm{ml} \mathrm{FZ}$ solution $(700 \mathrm{mg} / \mathrm{l})$ each day for 4,8 or 12 weeks (16). A pathophysiological study of the hearts was performed as previously described (17), following anesthetization of rats using intraperitoneal injections of $10 \%$ chloral hydrate $(30 \mu \mathrm{l} / \mathrm{kg}$; Beijing Dingguo Biotechnology Co., Ltd, Beijing, China). Two-dimensional transthoracic echocardiography was used to examine the left ventricular end-diastolic dimensions (LVEDD), left ventricular end-systolic dimensions (LVESD), fraction shortening (FS) and left ventricular ejection fraction (LVEF) (18).

Measurement of aortic pressure $(A O P)$ and right atrium pressure (RAP). AOP was measured as previously described (19), Four-dimensional phase contrast magnetic resonance imaging (ECG100C-MRI Electrocardiogram Amplifier; Biopac Systems, Inc., Goleta, CA, USA) was used for computation of the AOP waveform. The AOP waveform was then calculated using a segmentation algorithm. RAP was examined using the combination of 2-dimensional echocardiographic parameters and a 3-dimensional right atrial volume index (RA volume, $11 \pm 2.8 \mathrm{ml} / \mathrm{m}^{2}$ ) (20). The mean AOP and RAP were recorded.

Angiotensin (Ang) I, II and renin assay. Ang I and II concentration was measured using a modification of a previously described method (21). In brief, blood was collected from rats in a tube containing $2 \mathrm{mg} / \mathrm{ml} \mathrm{K}_{3}$ EDTA, $3 \mathrm{mM}$ 1,10-phenanthroline and $4 \mu \mathrm{M}$ enalaprilat, which were all purchased from Beijing Dingguo Biotechnology Co., Ltd. Samples were spiked with Iodine (I) ${ }^{125}$-Ang I and II (Beijing Dingguo Biotechnology Co., Ltd) as the internal controls. Samples were then analyzed using preconditioned C8 Bond Elute columns (GE Healthcare, Little Chalfont, UK). Angiotensins were eluted with $2 \mathrm{ml}$ water and acetonitrile (1:1; Beijing Dingguo Biotechnology Co., Ltd), and $0.1 \%$ trifluoroacetic acid (Beijing Dingguo Biotechnology Co., Ltd). The samples were then dried, resuspended in $20 \%$ acetonitrile/ $0.1 \%$ trifluoroacetic acid and filtered.

Filtered samples were purified using reverse-phase high-performance liquid chromatography with a C8 column (300 ̊, 4.6 mm internal diameter x $25 \mathrm{~cm}$; Rainin Dynamax, Woburn, MA, USA) at a rate of $1 \mathrm{ml} / \mathrm{min}$. Angiotensin peptides were separated by a linear gradient from $20-35 \%$ acetonitrile containing $0.1 \%$ trifluoroacetic acid. Retention times for angiotensins were as follows: Ang I, $16.5 \mathrm{~min}$; Ang II, 11.9 min; I $^{125}$-Ang I, $22 \mathrm{~min}$ and $\mathrm{I}^{125}$-Ang II, $16 \mathrm{~min}$. The purified fractions were dried, reconstituted with a buffer containing $10 \mathrm{mM}$ phosphate-buffered saline (PBS), $1 \mathrm{mM}$ EDTA, $0.25 \mathrm{mM}$ thimerasol and $0.1 \%$ gelatin $(\mathrm{pH} 7.3)$, which were all purchased from Beijing Dingguo Biotechnology Co., Ltd. Ang I and II were quantitatively measured using Angiotensin I and II ( $\left.{ }^{125} \mathrm{I}\right)$ Radioimmunoassay (RIA) kits (Amersham Plc, Amersham, UK). The RIA reaction mixture was incubated for $12 \mathrm{~h}$ and treated with dextran-coated charcoal (Beijing Dingguo Biotechnology Co., Ltd) to separate Ang I and II. Each RIA mixture was centrifuged at $1,500 \mathrm{x} \mathrm{g}$ at $4^{\circ} \mathrm{C}$ for $30 \mathrm{~min}$. The supernatant was counted and the Ang I and II values were read off a standard curve constructed using the solutions of known Ang concentrations. For renin activity assays, blood was collected in syringes containing $1.5 \mathrm{mg} / \mathrm{ml}$ EDTA and immediately centrifuged at $4^{\circ} \mathrm{C}$. Plasma was frozen in $1-\mathrm{ml}$ aliquots at $-20^{\circ} \mathrm{C}$ until further use. Renin was determined as previously described (22).

Immunohistochemistry. Three rats from each group were sacrificed following $30 \mathrm{~min}$ anaesthesia with sodium pentobarbitone $(50 \mathrm{mg} / \mathrm{kg}$; Sihuan Pharmaceutical Holdings Group Ltd., Beijing, China) by cervical dislocation at weeks 4, 8 or 12 . Immunohistochemistry was performed as previously described (23). In brief, paraffin-embedded heart tissues were sectioned into $2-\mu \mathrm{m}$ serial sections and then deparaffinized by incubating in xylene (Beijing Chemical Plant, Beijing, China), which was repeated four times for 5 min each; slides were then rehydrated by immersion in normal saline for $30 \mathrm{sec}$. Slides were treated with citric acid buffer ( $\mathrm{pH}$ 6.0; Beijing Dingguo Biotechnology Co., Ltd) with $0.2 \%$ Tween 20 (Beijing Dingguo Biotechnology Co., Ltd) and boiled for $10 \mathrm{~min}$ in order to retrieve the antigen. Slides were then rinsed in $10 \%$ $\mathrm{H}_{2} \mathrm{O}_{2}$ solution and blocked with methanol for $10 \mathrm{~min}$. Slides were incubated with mouse anti-human G9a monoclonal antibodies (1:1,000; Abcam, Cambridge, MA, USA) and mouse anti-human CAM monoclonal antibodies (1:1,000; Abcam) overnight at $4^{\circ} \mathrm{C}$. Samples were then rinsed in PBS and incubated for $1 \mathrm{~h}$ with secondary antibodies labeled with streptoavidin-biotin-peroxidase (Shanghai Sangon Biotechnology Co. Ltd., Shanghai, China). The bound complexes were then visualized using diaminobenzidine liquid chromogen and counterstaining with hematoxylin.

RNA interference. Primary neonatal cardiomyocytes (PNCs) from three-day-old Wistar rats, which were obtained from Beijing Vital River Laboratories, Co., Ltd (Beijing, China) were prepared as previously described (24). In order to inhibit G9a expression, small interfering (si)RNA targeting G9a (si-G9a) was used (Shanghai GeneChem Co. Ltd, Shanghai, China). The cardiomyocytes were grown in Dulbecco's modified Eagle's medium (DMEM; Beijing Dingguo Biotechnology Co., Ltd) supplemented with $10 \%$ fetal bovine serum (Beijing Dingguo Biotechnology Co., Ltd) and agitated at $10 \mathrm{~min}$ intervals. Transfection of G9a RNAi was performed using HiperFect (Qiagen, Limburg, Netherlands) with 3'-fluorescein isothiocyanate (FITC)-labeled control siRNA (AUAUAAUUGUGGCCGUUGCdTdT) or 3'-FITC-labeled si-G9a (GUCUCUACUAUGAUUCCUACUDTDT) according to the manufacturer's instructions. Following incubations of $36 \mathrm{~h}$, transfection efficiencies were determined using fluorescence microscopy (IX 50; Olympus, Tokyo, Japan). 
Table I. Analysis of multiple independent parameters of dilated cardiomyopathy.

\begin{tabular}{|c|c|c|c|}
\hline & Weeks & Control group & FZ-treated group \\
\hline \multirow[t]{4}{*}{ LVEDD (cm) } & 0 & $0.50 \pm 0.17$ & $0.50 \pm 0.15$ \\
\hline & 4 & $0.51 \pm 0.13$ & $0.59 \pm 0.12^{\mathrm{a}}$ \\
\hline & 8 & $0.53 \pm 0.22$ & $0.66 \pm 0.18^{\mathrm{b}}$ \\
\hline & 12 & $0.49 \pm 0.19$ & $0.69 \pm 0.20^{b}$ \\
\hline \multirow[t]{4}{*}{$\operatorname{LVESD}(\mathrm{cm})$} & 0 & $0.28 \pm 0.08$ & $0.27 \pm 0.06$ \\
\hline & 4 & $0.29 \pm 0.10$ & $0.35 \pm 0.11^{b}$ \\
\hline & 8 & $0.31 \pm 0.12$ & $0.39 \pm 0.12^{\mathrm{b}}$ \\
\hline & 12 & $0.33 \pm 0.15$ & $0.48 \pm 0.19^{b}$ \\
\hline \multirow[t]{4}{*}{ FS (\%) } & 0 & $45.51 \pm 4.10$ & $45.89 \pm 3.91$ \\
\hline & 4 & $46.63 \pm 4.51$ & $38.57 \pm 2.21^{\mathrm{b}}$ \\
\hline & 8 & $42.48 \pm 3.62$ & $30.17 \pm 1.18^{b}$ \\
\hline & 12 & $44.02 \pm 3.19$ & $28.74 \pm 1.48^{b}$ \\
\hline \multirow[t]{4}{*}{ LVEF (\%) } & 0 & $84.25 \pm 6.78$ & $84.62 \pm 5.81$ \\
\hline & 4 & $82.21 \pm 7.73$ & $79.12 \pm 3.87^{\mathrm{a}}$ \\
\hline & 8 & $83.96 \pm 5.98$ & $65.19 \pm 4.16^{\mathrm{b}}$ \\
\hline & 12 & $80.01 \pm 6.69$ & $60.99 \pm 3.39^{b}$ \\
\hline \multirow[t]{4}{*}{$\mathrm{AOP}(\mathrm{mmHg})$} & 0 & $84.06 \pm 6.12$ & $84.93 \pm 6.55$ \\
\hline & 4 & $89.45 \pm 7.18$ & $88.99 \pm 7.62$ \\
\hline & 8 & $90.45 \pm 5.69$ & $91.03 \pm 5.23$ \\
\hline & 12 & $88.87 \pm 5.23$ & $90.12 \pm 6.21$ \\
\hline \multirow[t]{4}{*}{ RAP (mmHg) } & 0 & $5.53 \pm 1.17$ & $5.78 \pm 2.15$ \\
\hline & 4 & $4.79 \pm 1.11$ & $13.77 \pm 4.42^{\mathrm{b}}$ \\
\hline & 8 & $6.47 \pm 1.01$ & $19.98 \pm 5.53^{b}$ \\
\hline & 12 & $5.81 \pm 1.36$ & $20.91 \pm 3.27^{b}$ \\
\hline \multirow[t]{4}{*}{ Renin } & 0 & $2.38 \pm 0.25$ & $2.35 \pm 0.19$ \\
\hline & 4 & $2.41 \pm 0.28$ & $3.61 \pm 0.28^{b}$ \\
\hline & 8 & $2.39 \pm 0.29$ & $3.45 \pm 0.65^{\mathrm{b}}$ \\
\hline & 12 & $2.35 \pm 0.21$ & $3.59 \pm 0.86^{\mathrm{b}}$ \\
\hline \multirow[t]{4}{*}{ Angiotensin I } & 0 & $17.07 \pm 2.28$ & $17.55 \pm 2.76$ \\
\hline & 4 & $16.89 \pm 1.97$ & $10.99 \pm 2.31^{\mathrm{b}}$ \\
\hline & 8 & $18.01 \pm 2.69$ & $11.69 \pm 3.24^{\mathrm{b}}$ \\
\hline & 12 & $16.63 \pm 2.43$ & $11.22 \pm 2.22^{\mathrm{b}}$ \\
\hline \multirow[t]{4}{*}{ Angiotensin II } & 0 & $65.13 \pm 7.99$ & $64.31 \pm 8.22$ \\
\hline & 4 & $69.52 \pm 9.11$ & $108.30 \pm 11.49^{b}$ \\
\hline & 8 & $70.14 \pm 7.95$ & $110.23 \pm 13.70^{\mathrm{b}}$ \\
\hline & 12 & $71.19 \pm 8.75$ & $113.56 \pm 15.77^{b}$ \\
\hline
\end{tabular}

${ }^{\mathrm{a}} \mathrm{P}<0.05$ and ${ }^{\mathrm{b}} \mathrm{P}<0.01$ vs. control group. LVEDD, left ventricular end-diastolic dimensions; LVESD, left ventricular end-systolic dimensions; FS, fraction shortening; LVEF, left ventricular ejection fraction; AOP, aortic pressure; RAP, right atrium pressure; FZ, furazolidone.

Reverse transcription quantitative polymerase chain reaction $(R T-q P C R)$. Hearts were excised in ice-cold $30 \mathrm{mM} \mathrm{KCl}$ (Beijing Dingguo Biotechnology Co., Ltd). Tissues and PNCs were snap-frozen in liquid nitrogen for RNA analysis. First-strand complementary (csNA was synthesized using the PrimeScript 1st strand cDNASynthesis kit (Takara Bio, Inc., Dalian, China). Primers specific to CAM forward, (5'-CCATC TACAACGCCAACATCG-3' and reverse, 5'-CACAATGACC TGAATGTCCTTG-3') and G9a cDNA forward, (5'-CCAATG ACACGTCTTCACTGG-3' and reverse, 5'-GTGGGGACA
GAAGACCATCCC-3') were used to detect the gene transcripts as described previously (25). GAPDH (forward, 5'-CGGAGTC AACGGATTTGGTCGTAT-3' and reverse, 5'-AGCCTT CTCCATGGTGGTGAAGAC-3') was used as a loading control. All primers were synthesized by Beijing BGI-GBI Biotech Co., Ltd (Beijing, China).

Western blot analysis. Grounded tissues and PNCs were lysed using a buffer [2\% SDS, $100 \mathrm{mM}$ Tris ( $\mathrm{pH}$ 6.8), $10 \%$ glycerol and $10 \mathrm{mM}$ dithiothreitol; Beijing Dingguo 


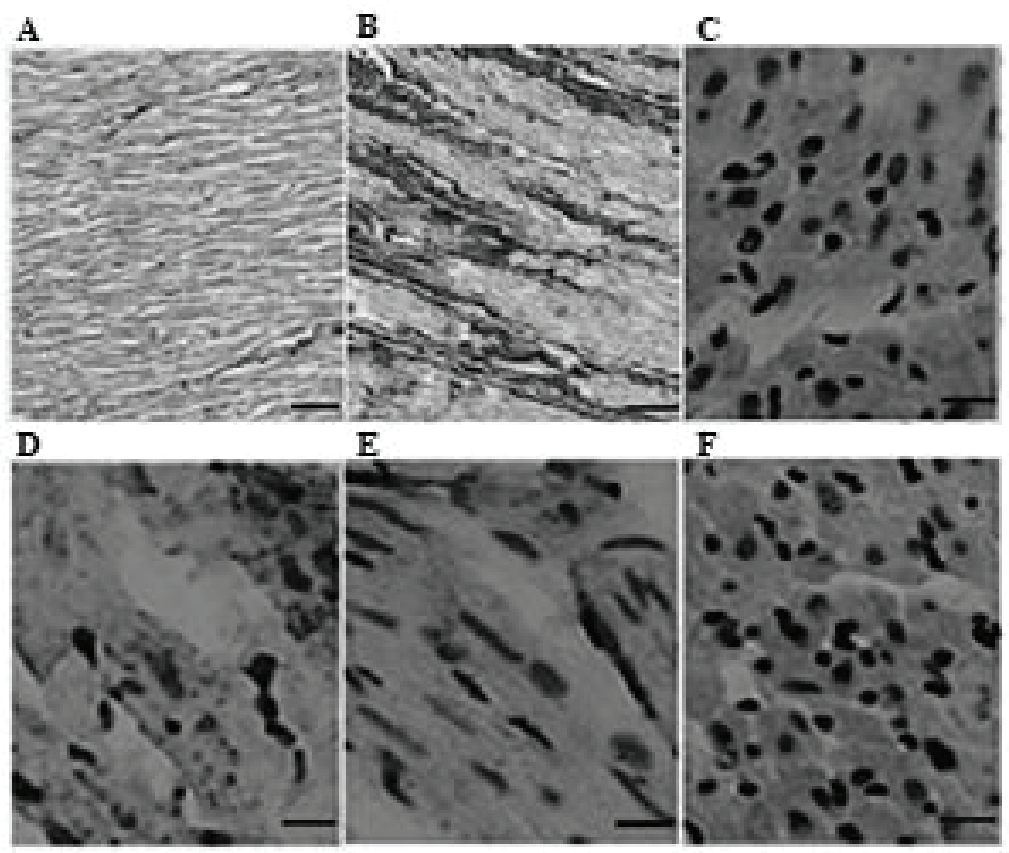

Figure 1. Immunohistochemical analysis of heart muscle characeristics and protein expression of CAM and G9a in DCM rat models. Characteristics of (A) normal heart muscle with low levels of fibrosis and (B) DCM model heart muscle with high levels of fibrosis. Protein expression of CAM in (C) normal heart muscle and (D) DCM model heart muscle. Protein expression of G9a in (E) normal heart muscle and (F) DCM model heart muscle. Scale bar, $50 \mu$ m; magnification, x200. CAM, cell adhesion molecules; G9a, H3K9 histone methyltransferase G9a; DCM, dilated cardiomyopathy.

Biotechnology Co., Ltd]. Samples were boiled and subjected to $10 \%$ SDS-PAGE (Beijing Dingguo Biotechnology Co., Ltd). Proteins were then transferred to polyvinylidene fluoride membranes (HMA-0200; NTR Systems, Seattle, WA, USA). Membranes were then immersed in 5\% non-fat milk in Tris-buffered saline buffer ( $\mathrm{pH}$ 7.6; Beijing Dingguo Biotechnology Co., Ltd). Blots were then incubated for $15 \mathrm{~min}$ with mouse anti-human monoclonal G9a antibody $(1: 1,000$; Abcam), followed by 30-min incubation with the secondary antibody alkaline-phosphatase-conjugated goat anti-mouse immunoglobulin G (1:1,000; Beijing Dingguo Biotechnology Co., Ltd). Molecular weights were determined using known markers (PageRuler Plus Prestained Protein Ladder; \#26619; Thermo Fisher Scientific, Beijing, China) and assays were performed in duplicate.

Evaluation of the growth rate of PNCs. PNCs were prepared from hearts of three-day-old Wistar rats as previously described (26). In brief, following isolation from non-myocyte fibroblasts via plating, PNCs were purified using a Percoll gradient, plated at a density of $0.5 \times 10^{5}$ cells/well in 12-well plates coated with $1 \%$ gelatin and grown in media (DMEM and Media 199, 4:1), supplemented with $2 \mathrm{mM}$ glutamine, $100 \mathrm{U} / \mathrm{ml}$ penicillin, $100 \mathrm{mg} / \mathrm{ml}$ streptomycin and $0.1 \mathrm{mmol} / 1 \mathrm{BrdUrd}$, which were all purchased from Beijing Dingguo Biotechnology Co., Ltd. BrdUrd was used to inhibit the growth of cardiac fibroblasts. The experimental group was treated with $10 \mathrm{pM} \mathrm{FZ}$ and the growth rate of PNCs was evaluated in the G9a RNAi and FZ-treated PNCs.

Statistical analysis. Statistical analysis was performed using SPSS 20.0 software (International Business Machines, Armonk, NY, USA). Values are presented as the mean \pm standard deviation. The Student's t-test was used to compare each variable between the DCM and control rats. $\mathrm{P}<0.05$ was considered to indicate as statistically significant difference between values.

\section{Results}

Cardiac function. Analysis of cardiac function in rats is challenging due to their small heart size and rapid heartbeat; therefore, the sensitivity of cardiac function measures is extremely low. In order to reduce the error in the present study, multiple parameters of DCM were analyzed and compared with those of wild-type rats. The total incidence rate of DCM in the experimental group was $80 \%$ (24/30). DCM is defined as the dilatation and impaired contractility of the LV; in addition, the LVEDD and LVESD contribute to the LV dilatation and cardiac dysfunction (27). In the present study, the LVEDD and LVESD were significantly increased in the FZ-treated group compared with those in the control group $(\mathrm{P}<0.05$ or $\mathrm{P}<0.01)$ (Table I). FS was used to measure the pump function of the heart; it was calculated using the ratio of the diameter of the left ventricle when it was relaxed and contracted, with normal values commonly observed to be $>26 \%$ (6). The LVEF represents the volumetric fraction of blood pumped out of the ventricle with each heart beat and may be applied to the left ventricle which ejects via the aortic valve into the systemic circulation (28). In the present study, the FS and LVEF values for the FZ-treated group were significantly decreased compared with those in the control group $(\mathrm{P}<0.01)$ (Table I). The AOP is the blood pressure at the root of the aorta, which was used to assess the efficacy of antihypertensive treatment with respect to cardiovascular risk factors (29). The RAP is the blood pressure in the thoracic vena cava, which was used as a major determinant of the right 
A
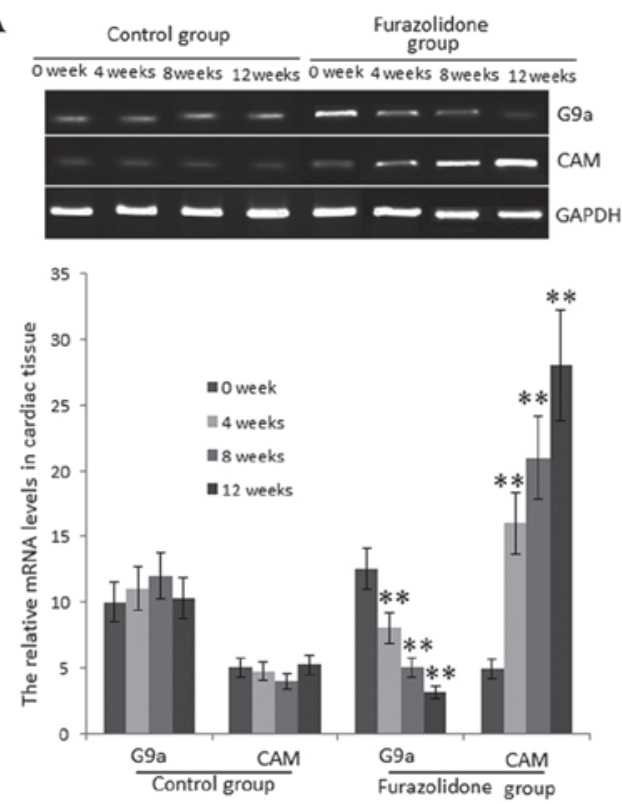
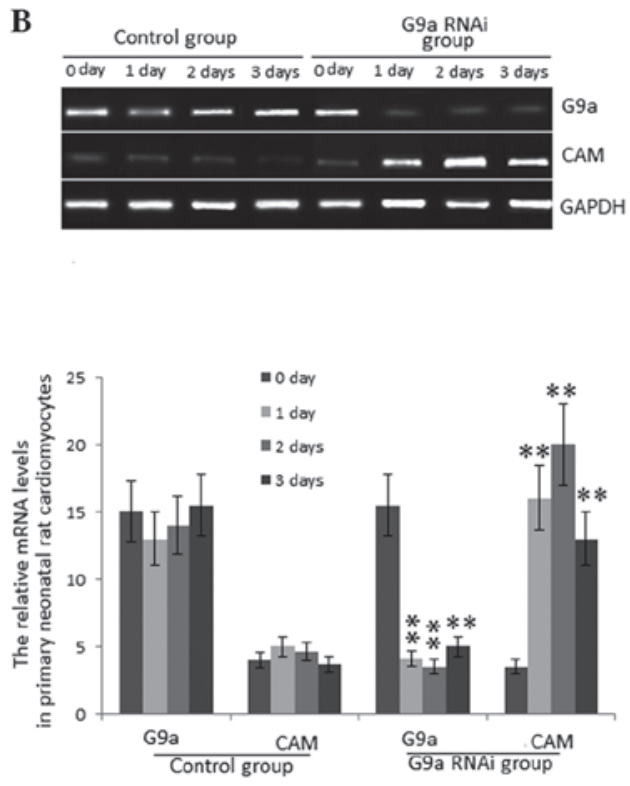

Figure 2. Reverse transcription polymerase chain reaction analysis of the relative mRNA levels of CAM and G9a. (A) Relative mRNA levels of CAM and G9a in DCM model and wild-type control rats at $0,4,8$ and 12 weeks of furazolidone treatment. (B) Relative mRNA levels of CAM and G9a in the PNCs and G9a RNAi-transfected PNCs at 0-3 days following transfection. Values are expressed as the mean \pm standard deviation. ${ }^{* *} \mathrm{P}<0.01$ vs. controls at day or week 0 . mRNA, messenger RNA; CAM, cell adhesion molecules; G9a, H3K9 histone methyltransferase G9a; DCM, dilated cardiomyopathy; PNCs; primary neonatal cardiomyocytes; RNAi, RNA interference.

A
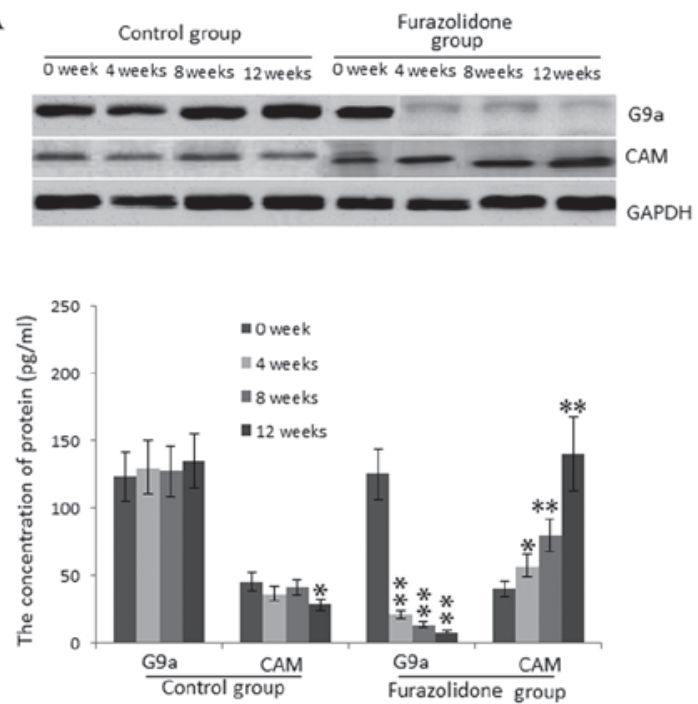

B
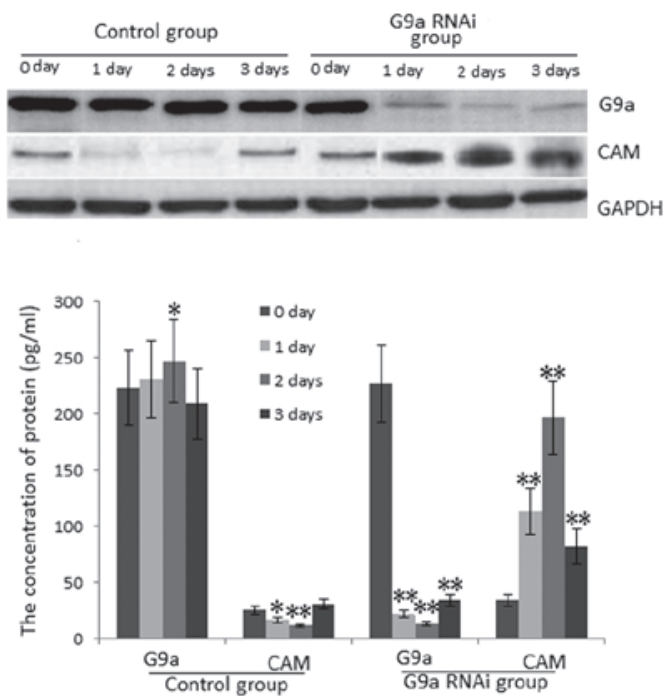

Figure 3. Western blot analysis of the relative protein levels of CAM and G9a. (A) Relative protein levels of CAM and G9a in DCM model and wild-type control rats at 0,4,8 and 12 weeks of furazolidone treatment. (B) Relative protein levels of CAM and G9a in the PNCs and G9a RNAi-transfected PNCs at $0-3$ days following transfection. Values are expressed as the mean \pm standard deviation. ${ }^{*} \mathrm{P}<0.05$ or ${ }^{* *} \mathrm{P}<0.01$ vs. controls at day or week 0 . mRNA, messenger RNA; CAM, cell adhesion molecules; G9a, H3K9 histone methyltransferase G9a; DCM, dilated cardiomyopathy; PNCs; primary neonatal cardiomyocytes; RNAi, RNA interference.

ventricular end diastolic volume (30). In the present study, the AOP was not significantly altered in the FZ-treated group compared with that in the control group $(\mathrm{P}>0.05)$ (Table I). However, compared with the control group, the RAP increased significantly in the FZ-treated group $(\mathrm{P}<0.01)$ (Table I).

Renin is an enzyme that participates in the renin-angiotensin system which mediates extracellular volume and arterial vasoconstriction, therefore regulating the body's mean arterial blood pressure (31). Angiotensin is a peptide hormone which causes vasoconstriction and an increase in blood pressure (32). Angiotensin also stimulates the release of aldosterone from the adrenal cortex, which promotes sodium retention in the distal nephron of the kidney and, in turn, increases the blood pressure (33). Angiotensin I is formed in the liver following the interaction of renin with $\alpha$-2-glycoprotein (34). Renin is produced in the kidneys in response to renal sympathetic activity, reduced intrarenal blood pressure or decreased delivery of $\mathrm{NaCl}$ to the macula densa (35). Angiotensin I is converted to 


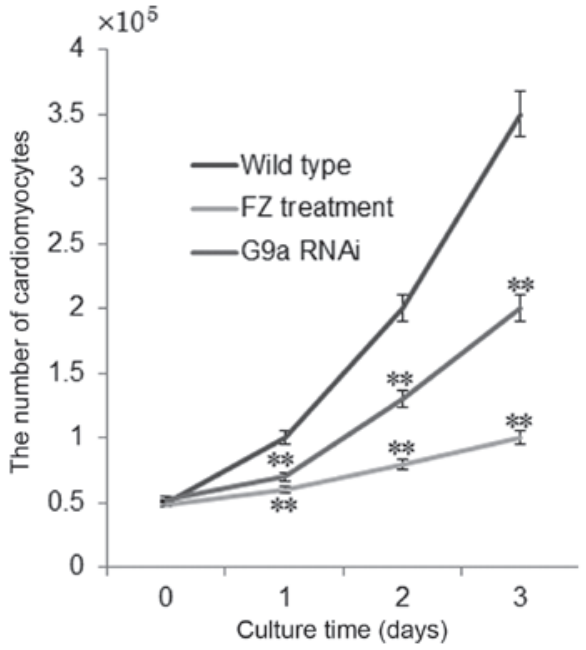

Figure 4. Effects of FZ treatment and G9a RNAi on the growth rate of primary neonatal cardiomyocytes at 0-3 days following transfection. Values are expressed as the mean \pm standard deviation. ${ }^{* *} \mathrm{P}<0.01$ vs. controls at day or week 0. FZ, furazolidone; G9a, H3K9 histone methyltransferase G9a; RNAi, RNA interference.

angiotensin II through the removal of two C-terminal residues by angiotensin-converting enzyme (36). Angiotensin II acts as an endocrine, autocrine/paracrine and intracrine hormone (37). In the present study, protein expression levels of renin, angiotensin I levels were markedly decreased, while angiotensin II levels were significantly increased in the FZ-treated group compared with those in the control group $(\mathrm{P}<0.01)$ (Table I).

Immunohistochemical analysis of the DCM model. As shown in Fig. 1A, the heart muscle of rats in the control group showed markedly less fibrosis compared with that in the experimental group (Fig. 1B), which was consistent with the characteristics of DCM (38). In addition, compared with that of the control group, CAM was highly expressed in the DCM muscles (Fig. 1C and D, respectively). Conversely, G9a expression was markedly higher in the control group compared with that in the DCM model (Fig. 1E and F, respectively). These results therefore suggested that G9a affected the expression levels of CAM in the development of DCM.

mRNA expression levels of G9a and CAM in the DCM model. G9a is a mammalian histone methyltransferase which contributes to the epigenetic silencing of tumor suppressor genes. Emerging evidence suggested that G9a was required in order to maintain a malignant phenotype (6). However, the biological function of G9a in DCM has remained to be elucidated. CAMs were reported to be higly expressed in DCM (10), whereas G9a repressed the expression of CAMs through CAM gene silencing. In order to explore the underlying molecular mechanisms of this in the present study, mRNA expression levels of G9a and CAM were investigated using RT-qPCR. The results showed that mRNA levels of G9a were significantly decreased in the FZ-treated group compared with those in the control group $(\mathrm{P}<0.01)$ (Fig. 2A). Conversely, mRNA levels of CAMs significantly increased in the $\mathrm{FZ}$-treated group $(\mathrm{P}<0.01)$ (Fig. 2A). In order to further determine the negative association of mRNA expression between G9a and CAM, the G9a gene was silenced in PNCs via G9a RNAi. As shown in Fig. 2B, mRNA levels of G9a significantly decreased following one-day RNAi compared with those in the control group. Conversely, mRNA levels of CAM significantly increased in PNCs in the G9a RNAi group $(\mathrm{P}<0.01)$ (Fig. 2B). This therefore indicated that G9a inhibited the progression of DCM through silencing the CAM gene.

Protein expression levels of G9a and CAM in DCM. DCM and wild-type control rats were sacrificed for analysis following 0 , 4,8 or 12 weeks of treatment. CAM and G9a expression in heart tissue was assessed using western blot analysis. Significantly decreased expression levels of G9a were observed in the cardiac tissues of DCM model rats compared with those in the control rats at 4, 8 and 12 weeks $(\mathrm{P}<0.01)$ (Fig. 3A). However, protein expression levels of CAM were significantly increased in the DCM model group compared with those in the control group at $4(\mathrm{P}<0.05), 8$ and 12 weeks $(\mathrm{P}<0.01)$ (Fig. 3A). Furthermore, significantly higher levels of CAM were found in PNCs transfected with G9a RNAi compared with those in the the wild-type PNC group (Fig. 3B). These results suggested that G9a inhibited the progression of DCM through repressing CAM expression.

Growth rate of PNCs. The growth rate of PNCs was significantly inhibited following treatment with FZ or G9a RNAi. Following three-day culture with FZ or G9a RNAi, the growth rate of PNCs was inhibited by 70 and $35 \%$, respectively, compared to that in the control group $(\mathrm{P}<0.01)$ (Fig. 4). The inhibitory mechanism of repression of PNC growth by FZ proceeds via the induction of apoptosis in cardiomyocytes (39). G9a RNAi reduced the growth inhibition of PNCs in FZ-induced DCM through silencing CAM gene expression, therefore enhancing the growth of cardiomyocytes.

\section{Discussion}

In the present study, in order to assess whether the DCM model was successfully constructed, multiple parameters of DCM were evaluated. The results showed that the reconstructed model exhibited all the parameters consistent with the characteristics of DCM compared with those of the wild-type rats. LVEDD, LVESD, RAP, renin and angiotensin II levels were significantly increased in DCM models compared with those in the wild-type animals, while angiotensin I levels were significantly decreased. Conversely, the parameters FS and LVEF were significantly decreased in DCM model rats. However, AOP was not significantly different between the DCM model and wild-type rats. These results therefore indicated that FZ successfully induced the DCM model in rats; these results were in concurrence with a previous study involving FZ-induced DCM in turkeys and rats (40). These models provided convenient methods for exploring the molecular mechanism for the risk factors and pathogenesis of DCM.

G9a histone methyltransferase has a dominant role in euchromatic histone H3 lysine-9 methylation, which is essential for early embryogenesis (41). G9a was reported to promote lung cancer invasion and metastasis through silencing the cell adhesion molecule gene Ep-CAM (6). G9a has also been found to inhibit the expression of numerous other genes (42); however, the biological function of G9a in DCM remains to be 
elucidated. The results of the present study demonstrated that G9a was expressed at low levels in DCM model rats compared with those of the control rats; this therefore indicated that G9a may decrease the risk of DCM through downregulating the expression of CAM. However, the precise role of G9a in the pathophysiology of DCM remains elusive, as previous studies have not defined whether altered expression levels of G9a in DCM were pathophysiologically important or simply an epiphenomenon associated with DCM (43). Furthermore, G9a is only one member of the histone H3 methyltransferases and numerous other members may also be associated with the development of DCM. However, G9a was found to repress the protein expression of CAM and may therefore inhibit the progression of DCM.

It may be of interest for future studies to explore the association of protein levels between cardiac CAMs and G9a in human heart failure, which may then be compared with the present results from DCM models of rats. The present study strongly suggested an important pathophysiological role for low levels of G9a in the development of DCM; in addition, the rat model used provided the opportunity to evaluate the mechanisms which initiated the expression of CAMs in the development of DCM. The DCM model develops a primary phenotype consistent with congestive heart failure, which resulted in cardiac dilatation and left ventricular dysfunction. This model allowed for the analysis of physiological and biochemical changes during development of end-stage heart failure, therefore providing a potential unique platform for the preclinical evaluation of G9a pharmacological therapies for inhibiting the progression of DCM.

In conclusion, the rat model of DCM was successfully induced through daily administration of FZ to rats. In the DCM-induced rats, the parameters for LVEDD, LVESD, RAP, renin as well as angiotensin I and II were significantly increased compared to those in the wild-type group, whereas FS and LVEF were significantly decreased in the DCM model, indicating that the rat model closely reflected the pathophysiological characters of DCM. The mRNA and protein expression levels of G9a were found to be significantly lower in the DCM model compared with those in the control group, while the expression levels of CAM were significantly increased in the DCM model. In PNCs, the expression of CAM was upregulated following G9a gene silencing by RNAi. These results indicated that G9a reduced the risk of DCM through downregulation of CAMs in a rat model of DCM; this therefore indicated the potential novel therapeutic use of G9a in the treatment of DCM.

\section{References}

1. Linke WA and Bücker S: King of hearts: a splicing factor rules cardiac proteins. Nat Med 18: 660, 2012.

2. Meyer L, Stubbs B, Fahrenbruch C, et al: Incidence, causes and survival trends from cardiovascular-related sudden cardiac arrest in children and young adults 0 to 35 years of age a 30 -year review. Circulation 126: 1363-1372, 2012.

3. Tachibana M, Sugimoto K, Fukushima T and Shinkai Y: Set domain-containing protein, G9a, is a novel lysine-preferring mammalian histone methyltransferase with hyperactivity and specific selectivity to lysines 9 and 27 of histone H3. J Biol Chem 276: 25309-25317, 2001.

4. Albert M and Peters AH: Genetic and epigenetic control of early mouse development. Curr Opin Genet Dev 19: 113-121, 2009.
5. Feldman N, Gerson A, Fang J, et al: G9a-mediated irreversible epigenetic inactivation of Oct-3/4 during early embryogenesis. Nat Cell Biol 8: 188-194, 2006.

6. Chen MW, Hua KT, Kao HJ, et al: H3K9 histone methyltransferase G9a promotes lung cancer invasion and metastasis by silencing the cell adhesion molecule Ep-CAM. Cancer Res 70: 7830-7840, 2010.

7. Devaux B, Scholz D, Hirche A, Klövekorn WP and Schaper J: Upregulation of cell adhesion molecules and the presence of low grade inflammation in human chronic heart failure. Eur Heart J 18: 470-479, 1997.

8. Blankenberg S, Rupprecht HJ, Bickel C, et al: Circulating cell adhesion molecules and death in patients with coronary artery disease. Circulation 104: 1336-1342, 2001.

9. Yin WH, Chen JW, Jen HL, et al: The prognostic value of circulating soluble cell adhesion molecules in patients with chronic congestive heart failure. Eur J Heart Fail 5: 507-516, 2003.

10. Noutsias M, Seeberg B, Schultheiss H-P and Kühl U: Expression of cell adhesion molecules in dilated cardiomyopathy evidence for endothelial activation in inflammatory cardiomyopathy. Circulation 99: 2124-2131, 1999.

11. Watanabe K, Ohta Y, Nakazawa M, et al: Low dose carvedilol inhibits progression of heart failure in rats with dilated cardiomyopathy. Br J Pharmacol 130: 1489-1495, 2000.

12. Chadfield MS and Hinton MH: In vitro activity of nitrofuran derivatives on growth and morphology of Salmonella enterica serotype enteritidis. J Appl Microbiol 96: 1002-1012, 2004.

13. Jankus E, Noren G and Staley N: Furazolidone-induced cardiac dilatation in turkeys. Avian Dis 16: 958-961, 1972.

14. Liao R, Nascimben L, Friedrich J, Gwathmey JK and Ingwall JS: Decreased energy reserve in an animal model of dilated cardiomyopathy relationship to contractile performance. Circ Res 78: 893-902, 1996.

15. Czarnecki CM: Animal models of drug-induced cardiomyopathy. Comp Biochem Physiol Part C 79: 9-14, 1984.

16. Huang RJ, Liu TW, Wu WF and Pang YS: Furazolidone induces dilated cardiomyopathy in rats [J]. Chinese J Pathophysiol 11, 2005.

17. Kubota T, McTiernan CF, Frye CS, et al: Dilated cardiomyopathy in transgenic mice with cardiac-specific overexpression of tumor necrosis factor- $\alpha$. Circ Res 81: 627-635, 1997.

18. Stein AB, Tiwari S, Thomas P, et al: Effects of anesthesia on echocardiographic assessment of left ventricular structure and function in rats. Basic Res Cardiol 102: 28-41, 2007.

19. Delles M, Rengier F, Jeong YJ, et al: Estimation of aortic pressure waveforms from 4D phase-contrast MRI. Conf Proc IEEE Eng Med Biol Soc 2013: 731-734, 2013.

20. Patel AR, Alsheikh-Ali AA, Mukherjee J, et al: 3D echocardiography to evaluate right atrial pressure in acutely decompensated heart failure correlation with invasive hemodynamics. JACC Cardiovasc Imaging 4: 938-945, 2011.

21. Goldberg MR, Tanaka W, Barchowsky A, et al: Effects of losartan on blood pressure, plasma renin activity and angiotensin II in volunteers. Hypertension 21: 704-713, 1993.

22. Kodish ME and Katz FH: Plasma renin concentration: comparison of angiotensinase inhibitors and correlation with plasma renin activity and aldosterone. J Lab Clin Med 83: 705-715, 1974.

23. Takano S, Yoshitomi H, Togawa A, et al: Apolipoprotein C-1 maintains cell survival by preventing from apoptosis in pancreatic cancer cells. Oncogene 27: 2810-2822, 2008.

24. Guo Z, Wang S, Jiao Q, Xu M and Gao F: RNAi targeting ryanodine receptor 2 protects rat cardiomyocytes from injury caused by simulated ischemia-reperfusion. Biomed Pharmacother 64: 184-190, 2010.

25. Carleton KL: Quantification of transcript levels with quantitative RT-PCR. Methods Mol Biol 772: 279-295, 2011.

26. Purcell NH, Tang G, Yu C, Mercurio F, DiDonato JA and Lin A: Activation of NF-kappa B is required for hypertrophic growth of primary rat neonatal ventricular cardiomyocytes. Proc Nat Acad Sci USA 98: 6668-6673, 2001.

27. Moon C, Krawczyk M, Ahn D, et al: Erythropoietin reduces myocardial infarction and left ventricular functional decline after coronary artery ligation in rats. Proc Nat Acad Sci 100: 11612-11617, 2003.

28. Paulus WJ, Tschöpe C, Sanderson JE, et al: How to diagnose diastolic heart failure: a consensus statement on the diagnosis of heart failure with normal left ventricular ejection fraction by the heart failure and echocardiography associations of the european society of cardiology. Eur Heart J 28: 2539-2550, 2007.

29. Avolio A: Central aortic blood pressure and cardiovascular risk a paradigm shift? Hypertension 51: 1470-1471, 2008. 
30. Bombardini T, Cini D, Arpesella G and Picano E: WEB downloadable software for training in cardiovascular hemodynamics in the (3-D) stress echo lab. Cardiovasc Ultrasound 8: 48, 2010.

31. Vander AJ: Control of renin release. Physiol Rev 47: 359-382, 1967.

32. Gigante B, Rubattu S, Russo R, et al: Opposite feedback control of renin and aldosterone biosynthesis in the adrenal cortex by angiotensin II AT1-subtype receptors. Hypertension $30(3 \mathrm{Pt} 2)$ : 563-568, 1997.

33. Sarafidis PA and Ruilope LM: Aggressive blood pressure reduction and renin-angiotensin system blockade in chronic kidney disease: time for re-evaluation? Kidney Int 85: 536-546, 2014.

34. Podwysocki B, Simon K and Gladysz A: Activity of angiotensin converting enzyme I and levels of acid alpha glycoprotein in selected liver and biliary tract diseases. Pol Tyg Lek 48: 268-270, 1993 (In Polish)

35. Aoyagi T, Izumi Y, Hiroyama M, et al: Vasopressin regulates the renin-angiotensin-aldosterone system via V1a receptors in macula densa cells. Am J Physiol Renal Physiol 295: F100-F107, 2008.

36. Erdös EG and Skidgel RA: Renal metabolism of angiotensin I and II. Kidney Int Suppl 30: S24-S27, 1990.

37. Johnston CI: Renin-angiotensin system: a dual tissue and hormonal system for cardiovascular control. J Hypertens 10: S27, 1992.
38. Ohtani K, Yutani C, Nagata S, Koretsune Y, Hori M and Kamada T: High prevalence of atrial fibrosis in patients with dilated cardiomyopathy. J Am Coll Cardiol 25: 1162-1169, 1995.

39. Zhang M, Shan H, Zhu Y-h, Lin L and Wei J: Attenuating effect of estrogen on furazolidone induced cardiomyocyte apoptosis is dependent on p66shc adapter protein. In: cardiology karger allschwilerstrasse 10, ch-4009 Basel, Switzerland: pp 34-34, 2013.

40. Genao A, Seth K, Schmidt U, Carles M and Gwathmey JK: Dilated cardiomyopathy in turkeys: an animal model for the study of human heart failure. Lab Anim Sci 46: 399-404, 1996.

41. Tachibana M, Sugimoto K, Nozaki M, et al: G9a histone methyltransferase plays a dominant role in euchromatic histone $\mathrm{H} 3$ lysine 9 methylation and is essential for early embryogenesis. Genes Dev 16: 1779-1791, 2002.

42. Roopra A, Qazi R, Schoenike B, Daley TJ and Morrison JF: Localized domains of G9a-mediated histone methylation are required for silencing of neuronal genes. Mol Cell 14: 727-738, 2004.

43. Hohl M, Wagner M, Reil JC, et al: HDAC4 controls histone methylation in response to elevated cardiac load. J Clin Invest 123: 1359-1370, 2013 